\title{
Recombinant human secretory leukocyte protease inhibitor ameliorated vessel preservation in experimentally isolated rat arteries
}

\author{
Kantapich Kongpol ${ }^{1,2}$, Rungrueang Yodsheewan ${ }^{3}$, Nitirut Nernpermpisooth ${ }^{1,4}$, Sarawut Kumphune S $^{1,5^{*}}$ \\ ${ }^{1}$ Biomedical Research Unit in Cardiovascular Sciences (BRUCS), Faculty of Allied Health Sciences, Naresuan University, Phitsanulok, 65000, Thailand. \\ ${ }^{2}$ Graduate program in Biomedical Sciences, Faculty of Allied Health Sciences, Naresuan University, Phitsanulok, Thailand. \\ ${ }^{3}$ Department of Pathology, Faculty of Veterinary Medicine, Kasetsart University, Kamphaeng Saen Campus 73140, Thailand. \\ ${ }^{4}$ Department of Cardio-Thoracic Technology, Faculty of Allied Health Sciences, Naresuan University, Phitsanulok, 65000, Thailand. \\ ${ }^{5}$ Department of Medical Technology, Faculty of Allied Health Sciences, Naresuan University, Phitsanulok, 65000, Thailand.
}

\section{ARTICLE INFO \\ Received on: 24/04/2020 \\ Accepted on: 31/06/2020 \\ Available online: 05/09/2020}

\section{Key words:}

Secretory leukocyte protease

inhibitor, preservative

solutions, vessel graft,

ischemia/reperfusion,

vascular reconstruction.

\begin{abstract}
Vessel allograft preservation is essential for vascular reconstruction surgery. The preservation solution is crucial in extending the preservation period while maintaining vascular endothelial function. Currently, a $0.9 \%$ normal saline solution (NSS) is widely used as a storage solution although its protective effect on endothelial preservation is limited. In this study, we determine the beneficial effect of recombinant human secretory leukocyte protease inhibitor (rhSLPI), supplemented to $0.9 \%$ NSS, for isolated aortic preservation. The thoracic and abdominal aorta were isolated from Wistar rats $(n=6)$, and the aortic rings were subsequently cut and preserved in $0.9 \%$ NSS in the presence and absence of $1 \mu \mathrm{g} / \mathrm{ml} \mathrm{rhSLPI}$ for $0,6,24$, and 48 hours. The preservative solution was collected to determine the released lactate dehydrogenase (LDH) activity and pro-inflammatory cytokine levels, including tumor necrosis factor $\alpha(\mathrm{TNF}-\alpha)$ and interleukin-6 (IL-6). Furthermore, the appearance and severity of vessel degeneration were subjected to blind histopathological assessment by pathologists. The results indicated that $0.9 \%$ NSS, supplemented with rhSLPI, significantly reduced the released LDH activity, TNF- $\alpha$, and IL-6 levels and could attenuate endothelial detachment, endothelial degeneration, and endothelial necrosis. This study demonstrated for the first time that adding rhSLPI to a saline preservative solution could prevent vascular injury and possibly extend the graft storage duration before clinical intervention.
\end{abstract}

\section{INTRODUCTION}

Vascular reconstruction is a therapeutic technique used in reconstructive arterial surgery for several pathological conditions including organ substitute, coronary artery bypass grafting in an ischemic heart (Ben Ali et al., 2018; Fahner et al., 2006; Kazemi et al., 2018; Wille et al., 2008), and infrarenal aortic replacement (Kieffer et al., 2004; Zatschler et al., 2009). A vascular allograft is the most attractive option for revascularization. However, the major challenge in transplantation is reducing vascular endothelial injury, which can promote inflammation following thrombosis-

\section{"Corresponding Author}

Sarawut Kumphune, Biomedical Research Unit in Cardiovascular Sciences (BRUCS), Faculty of Allied Health Sciences, Naresuan

University, Phitsanulok,65000,Thailand.E-mail: sarawutk@nu.ac.th mediated vascular graft failure (Ben Ali et al., 2018; Wille et al., 2008). To minimize the vessel injury, the protective strategies should be implemented immediately after meticulous graft harvesting with an appropriate preservation solution. The latter is a critical factor that can influence the clinical outcomes of longterm graft storage (Ben Ali et al., 2018).

Several organ preservative solutions such as the University of Wisconsin (UW) solution, histidine-tryptophanketoglutarate (HTK) solution, and Celsior solution have been used in vascular transplantations (Kazemi et al., 2018). Cold 0.9\% normal saline solution (NSS) is another preservative solution, which is used in some resource-limited (developing) countries (Kazemi et al., 2018). However, several studies have shown that NSS has a lower preservative efficacy when compared to other preservative solutions (Kazemi et al., 2018; Zatschler et al., 2009). Therefore, the addition or supplementation of a solution that could 
possibly enhance the preservative efficiency of NSS and prolong the vessel graft storage should be considered.

The secretory leukocyte protease inhibitor (SLPI) is a serine protease selective inhibitor that counteracts with an excessive inflammatory response (Majchrzak-Gorecka et al., 2016). The previous studies showed that adding recombinant human SLPI (rhSLPI) in a heart preservative solution could restore myocardial contraction (Schneeberger et al., 2008). Besides, the previous studies showed that the treatment of rhSLPI in human umbilical vein endothelial cells could reduce cell damage and inflammation, prevent death, and preserve cellular cytoskeleton integrity from in vitro I/R injury (Nernpermpisooth et al., 2017; Paiyabhroma et al., 2018; Prompunt et al., 2018a, 2018b). This suggests the efficacious cytoprotective effect of rhSLPI on vascular endothelial cells.

The previous study has also demonstrated that endothelialderived rhSLPI not only reduces endothelial cell injury but also attenuates cardiomyocyte death in an in vitro ischemia/reperfusion model (Kongpol et al., 2019). Therefore, the in vitro endothelial protective effect of rhSLPI could provide basic information for the implementation of vascular tissue. However, the effect of rhSLPI supplemented to $0.9 \% \mathrm{NSS}$ on vessel graft preservation has never been investigated. Therefore, we hypothesized that $0.9 \%$ NSS preservative solution, supplemented with rhSLPI, could prevent isolated rat aorta injury and prolong storage duration.

\section{MATERIALS AND METHODS}

\section{Experiment animals}

Adult male Wistar rats weighing 250-300 g $(n=6)$ were purchased from Nomura Siam International, Bangkok, Thailand. All animals were maintained under temperature $\left(22 \pm 1^{\circ} \mathrm{C}\right)$ with 12-hours light:dark cycle at the Center for Animal Research, Naresuan University, Phitsanulok, Thailand. All protocols used in this study were approved by the Animal Use and Care Committee at Naresuan University (NU-AE601129) and conformed to the guidelines set by the American Physiological Society and Animal Welfare Act.

\section{Isolation of rat aorta}

Rats were anesthetized by intraperitoneal injection of pentobarbital $(100 \mathrm{mg} / \mathrm{kg})$ and heparin (150 units). The deep anesthesia was closely observed and confirmed by a lack of both toe pinch and corneal reflexes. After that, the hearts were rapidly isolated. The thoracic and abdominal aorta were then excised quickly and placed in ice-cold NSS. The aorta was carefully cleaned from adjacent fatty tissue and cut into ring segments with $3-5 \mathrm{~mm}$ in length. Therefore, a total of 16 aortic rings per animal were incubated in $0.9 \% \mathrm{NSS}$ in the presence and absence of $1 \mu \mathrm{g} /$ $\mathrm{ml}$ rhSLPI and stored at $4{ }^{\circ} \mathrm{C}$ for various preservative periods $(0$, 6,24 , and 48 hours). One set of aortic rings, which preserved with and without rhSLPI at each period, was used for histopathological study. Another set of aortic rings was homogenized to collect protein and determined for the inflammatory cytokines by enzymelinked immunosorbent assay (ELISA).

\section{Vascular histopathology}

The histopathology technique used in this study was modified from Howat WJ and Wilson BA (Howat and Wilson, 2014). At the end of each preservation period, the aortic rings were fixed with $2.5 \%(\mathrm{v} / \mathrm{v})$ glutaraldehyde for 24 hours. Then, the rings were further fixed with $10 \%(\mathrm{v} / \mathrm{v})$ formalin pending a histopathological process.
Briefly, the rings were dehydrated, embedded into paraffin, and cut into 5-10- $\mu \mathrm{m}$-thick sections (PFM Rotary, Cologne, Germany). Then, the sections were stained with hematoxylin and eosin (H\&E) for histopathological analysis. All histopathological examinations were performed by an experienced pathologist. The pathologist was blind toward the experiment group, and the examination and scoring of vascular pathology were performed under a light microscope (Olympus). The identification of vascular endothelial cells was based on the information that the endothelial cells located at tunica intima showed as a single layer of squamous-to-fusiform shape cells close to the lumen of the blood vessel. In H\&E staining, the endothelial cells reveal as a flat layer of squamous-to-fusiform shape cells characterized by basophilic oval-shaped nucleus, which may protrude into the lumen of the blood vessel, and very few of eosinophilic cytoplasm. The vascular histopathology scoring criteria consisted of endothelial detachment, elastic membrane disruption, necrosis, endothelial degeneration/edema, and complete denudation (Kazemi et al., 2018). The severity score was graded as follows: grade $0=$ no lesion, grade $1=$ lesion less than $25 \%$ of tissue, and grade $2=$ lesion more than $25 \%$ of tissue.

\section{Determination of released lactate dehydrogenase (LDH) activity}

At the end of each preservative period, the preservation solution was collected and stored at $-20^{\circ} \mathrm{C}$ until analysis (Prompunt et al., 2018a). The released LDH activity was determined from the collected solutions using the LDH activity assay kit, which is a modified method based on the recommendations of the Scandinavian Committee on Enzymes (LDH SCE mod.). The kit was purchased from HUMAN (Wiesbaden, Germany). About 10 $\mu \mathrm{l}$ of collected preservative at each period was mixed with $1 \mathrm{ml}$ of reaction buffer and incubated at $37^{\circ} \mathrm{C}$ for 5 minutes. Then, $250 \mu \mathrm{l}$ of substrate reagent was added. The solution was mixed, and the absorbance was read at $\lambda 340 \mathrm{~nm}$. The mean absorbance change per minute ( $\triangle A /$ minute) was used for calculating LDH activity with the following formula:

$$
\text { LDH activity }(\mathrm{U} / \mathrm{I})=\Delta A / \text { minute } \times 20,000
$$

\section{Tissue homogenization and protein extraction}

Aortic rings, which preserved in NSS in the presence and absence of rhSLPI at each period, were snapped in liquid nitrogen and transferred to $-20^{\circ} \mathrm{C}$ freezer until analysis. About $50 \mathrm{mg}$ of aortic ring sample was homogenized in $500 \mu$ of homogenization buffer $\left[20 \mathrm{mM}\right.$ Tris $\mathrm{HCl}(\mathrm{pH} 6.8), 1 \mathrm{mM} \mathrm{Na}_{3} \mathrm{VO}_{4}$, and $5 \mathrm{mM}$ $\mathrm{NaF}$ ] using a mortar and pestle (Mongkolpathumrat et al., 2019). Then, the tissue homogenate was centrifuged at 14,000 rpm for 10 minutes at $4{ }^{\circ} \mathrm{C}$. The supernatant was collected for further experiments.

\section{Determination of protein concentration by Bradford assay}

The total protein concentration of tissue homogenate was measured by Bradford protein assay reagent (BIO-RAD, Hercules, CA) (Mongkolpathumrat et al., 2019). About $50 \mu \mathrm{l}$ of the protein samples were added into $2.5 \mathrm{ml}$ of Bradford reagent (BIO-RAD, Hercules, CA) and incubated at room temperature for at least 5 minutes. The absorbance was measured using a spectrophotometer at $\lambda 595 \mathrm{~nm}$. The relative protein concentration was calculated by the equation for the line generated in the Bovine Serum Albumin (BSA) standard curve. The protein concentration 
was used to adjust the amount of protein used for the determination of inflammatory cytokine by ELISA.

\section{Determination of inflammatory cytokines by ELISA}

ELISA was performed using a 2,2'-azinobis-(3ethylbenzothiazoline-6-sulfonate) (ABTS) ELISA Buffer Kit, Prepotech ${ }^{\circledR}$ (Mongkolpathumrat et al., 2019). The ELISA reagents were prepared at room temperature by gentle mixing. First, the precoated step was performed in 96-well plate overnight. On the following day, the plate was inverted to remove the liquid and blot the paper towel. After that, the plate was washed four times using $200 \mu \mathrm{l}$ of washing buffer solution. About $200 \mu \mathrm{l}$ of the blocking solution was added into the well and then incubated for 1 hour. Tissue homogenates $(10 \mu \mathrm{g}$ of protein) were added into the wells and incubated at room temperature for at least 2 hours followed by washing 4 times again. Then, the detection antibody was added and incubated at room temperature for 2 hours. The plate was then washed again, and $100 \mu \mathrm{l}$ of avidin-horseradish peroxidase conjugated 1:2,000 was added and incubated at room temperature for 30 minutes. After that, the ABTS liquid substrate was added and incubated at room temperature until the color was developed. The absorbance was measured using a spectrophotometer at $\lambda 405 \mathrm{~nm}$.

\section{Statistical analysis}

Statistical analysis was performed using commercially available software (GraphPad Prism version 7). All data were expressed as mean \pm S.E.M. All comparisons were assessed for significance using an unpaired $t$-test or analysis of variance (ANOVA) followed by the Tukey-Kramer test or Chi-square test when appropriate. A $p$-value less than 0.05 was considered to be statistically significant.

\section{RESULTS}

\section{Protective effect of rhSLPI decrease tissue injury by reduced LDH activity}

The aortic rings were preserved in cold NSS in the presence and absences of $1 \mu \mathrm{g} / \mathrm{ml}$ of rhSLPI for $0,6,24$, and 48 hours. Then, the preservative solution was collected to determine the released LDH activity. The results showed that there was a significant increase in released LDH activity in preservative solution at 6-48 hours when compared to control 0 hour (6 hours: $32.25 \pm 1.80 \mathrm{IU} / \mathrm{ml}, 24$ hours: $42.37 \pm 2.40 \mathrm{IU} / \mathrm{ml}$, and 48 hours: $77.58 \pm 1.60 \mathrm{IU} / \mathrm{ml} v s .0 .5 \pm 0.29 \mathrm{IU} / \mathrm{ml}, p<0.05)$. Moreover, the results showed that the released LDH activity in NSS with rhSLPI for 48 hours was significantly lower than that of NSS $(61.75 \pm$ $1.58 \mathrm{IU} / \mathrm{ml} v s .77 .58 \pm 1.60 \mathrm{IU} / \mathrm{ml}, p<0.05)$. However, there was a trend of the reduction of released LDH activity in NSS with rhSLPI at 6 and 24 hours but was not statistically significant when compared to NSS group (6 hours: $32.25 \pm 1.80 \mathrm{IU} / \mathrm{ml}$ and 6 hours with rhSLPI: $25.86 \pm 2.33 \mathrm{IU} / \mathrm{ml}, p>0.05$, and 24 hours: 42.37 $\pm 2.40 \mathrm{IU} / \mathrm{ml}$ and 24 hours with rhSLPI: $33.59 \pm 2.50 \mathrm{IU} / \mathrm{ml}, p>$ 0.05 ) (Fig. 1).

\section{Protective effect of rhSLPI decrease inflammatory cytokines}

The protein extract from aortic rings was used to determine the inflammatory cytokines by ELISA, including TNF- $\alpha$ and IL- 6 . The results showed that vessels preserved for 48 hours in both the groups significantly increased TNF- $\alpha$ level when compared to control (NSS: $338.9 \pm 50.15 \mathrm{pg} / \mathrm{ml}$, NSS with rhSLPI:

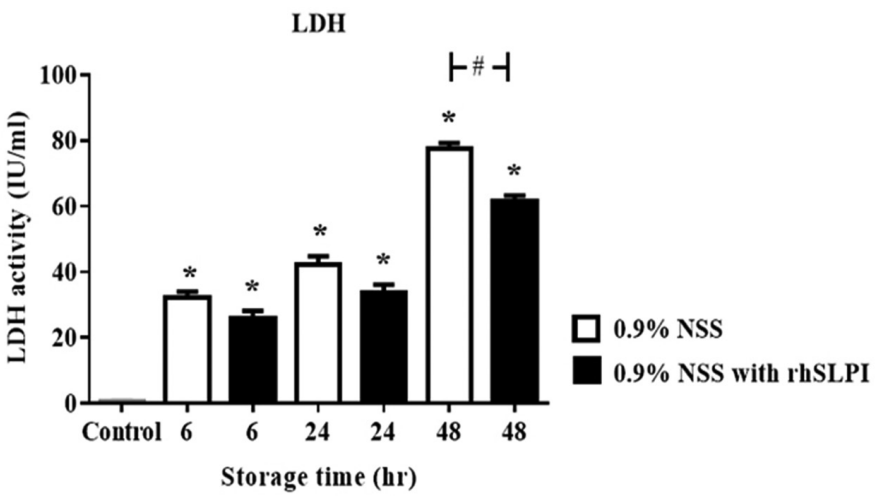

Figure 1. Determination of vascular injury by a released LDH activity in the preservative solution. Aortic rings were preserved in $0.9 \% \mathrm{NSS}$ or $0.9 \%$ NSS with rhSLPI preservative solution for $0,6,24$, and 48 hours, after which the solution was collected and assessed for LDH activity. Each bar graph represents the means \pm S.E.M. ${ }^{*} p<0.05$ versus storage at 0 hour (control) (ANOVA), $\# p<0.05$ versus among storage in $0.9 \%$ NSS, with or without rhSLPI.

$237 \pm 21.98 \mathrm{pg} / \mathrm{ml} v s$. control: $178 \pm 19.03 \mathrm{pg} / \mathrm{ml}, p<0.05)$. On the contrary, vascular preserved for 24 hours did not appear to be different when compared to control (NSS: $181.4 \pm 6.66 \mathrm{pg} / \mathrm{ml}$ and NSS with rhSLPI: $150.3 \pm 5.53 \mathrm{pg} / \mathrm{ml} v s$. control: $178 \pm 19.03 \mathrm{pg} /$ $\mathrm{ml}, p>0.05)$. However, the TNF- $\alpha$ level in the vessel preserved in NSS with rhSLPI for 24 hours was significantly lower than vascular preserved in NSS $(150.3 \pm 5.53 \mathrm{pg} / \mathrm{ml}$ vs. $181.4 \pm 6.66$ $\mathrm{pg} / \mathrm{ml}, p<0.05$ ) (Fig. 2A). Besides, the level of TNF- $\alpha$ in NSS with rhSLPI for 48 hours was lower than $0.9 \%$ NSS without rhSLPI but could not reach statistical significance $(237 \pm 21.98 \mathrm{pg} /$ $\mathrm{ml}$ vs. $338.9 \pm 50.15 \mathrm{pg} / \mathrm{ml}, p>0.05)$ (Fig. 2A).

Similarly, the vessels preserved for only 48 hours in both the groups showed a significant increase in IL-6 level when compared to control (NSS: $401.6 \pm 31.81 \mathrm{pg} / \mathrm{ml}$ and NSS with rhSLPI: $298.6 \pm 21.88 \mathrm{pg} / \mathrm{ml} v s$. control: $193.7 \pm 8.75 \mathrm{pg} / \mathrm{ml}, p$ $<0.05)$. Furthermore, the vessel preserved in NSS supplemented with rhSLPI for 24 hours was shown to be significantly lower when compared to control $(139.7 \pm 11.13 \mathrm{pg} / \mathrm{ml} v s .193 .7 \pm 8.75 \mathrm{pg} / \mathrm{ml}$, $p<0.05$ ). The IL-6 level vessels preserved in NSS supplemented with rhSLPI for 24 and 48 hours were shown to be significantly lower than that of NSS (24 hours: $139.7 \pm 11.13 \mathrm{pg} / \mathrm{ml} v \mathrm{~s} .180 .7 \pm$ $16.17 \mathrm{pg} / \mathrm{ml}, p<0.05$, and 48 hours: $298.6 \pm 21.88 \mathrm{pg} / \mathrm{ml} v s .401 .6$ $\pm 31.81, p<0.05)$ (Fig. 2B).

\section{Protective effect of rhSLPI on vascular histopathology}

Vascular histopathology was performed in both aortic tissues preserved in NSS and NSS supplemented with rhSLPI. The endothelial detachment, elastic membrane disruption, necrosis, endothelial degeneration/edema, and complete denudation were divided into no change or change. The results showed that there was no significant difference in the number of vessels with the presence of histopathological changes during different durations of preservation (Table 1).

To determine the severity of vascular histopathology as well as the effect of rhSLPI to preserve vascular integrity, the severity of endothelial detachment, elastic membrane disruption, necrosis, endothelial degeneration/edema, and complete denudation were graded on a scale ranging from 0 to 2 (Fig. 3).

For endothelial detachment, the results found that the vessels preserved in NSS with rhSLPI for 6 and 24 hours showed a 
A

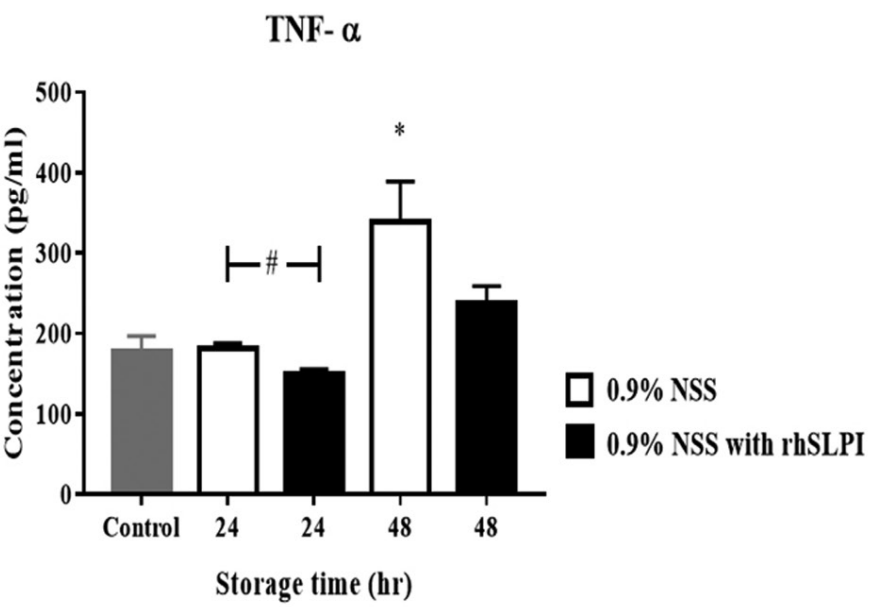

B

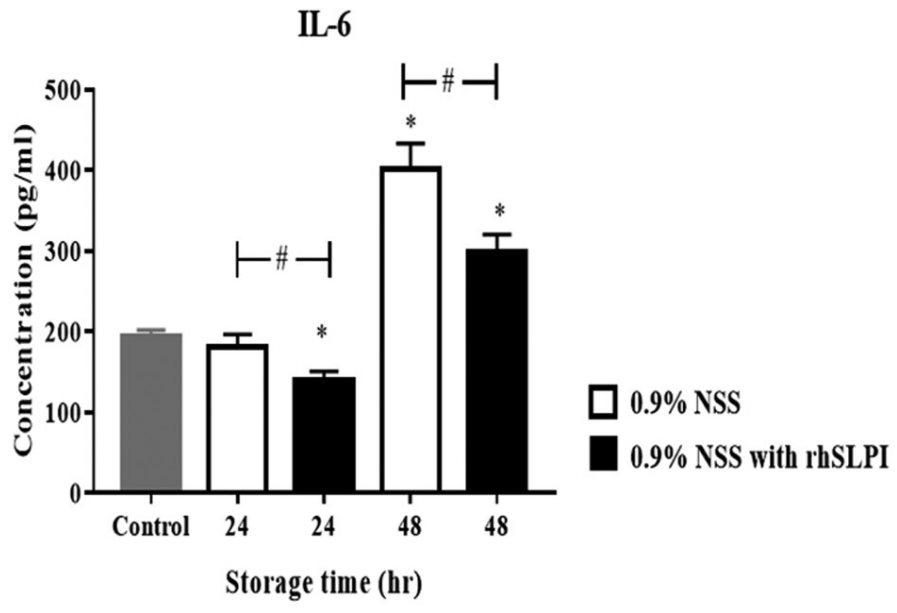

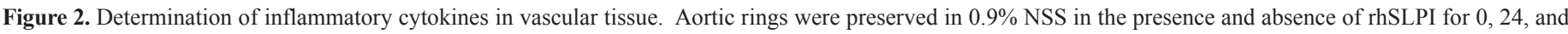

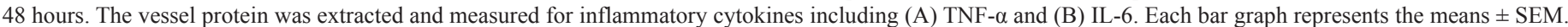
$* p<0.05$ versus storage at 0 hour (ANOVA), $\# p<0.05$ versus among storage in $0.9 \%$ NSS, with or without rhSLPI (ANOVA, $n=6$ ).

significantly lower endothelial detachment score when compared to the other groups (Fig. 4A). On the contrary, the vessels preserved in NSS with rhSLPI for 48 hours did not show any significant difference (Fig. 4A).

Similarly, the vessels preserved in NSS with rhSLPI for 6 and 24 hours showed a significantly lower elastic membrane disruption score when compared to the vessels preserved in NSS (Fig. 4B). Although the elastic membrane disruption score of the vessels preserved in NSS with rhSLPI for 48 hours was not significantly lower than other groups, the score was shown to have a lower trend in the vessels preserved in NSS with rhSLPI.

The necrosis scores for the vessels preserved in both groups of preservatives for 6 hours were not shown to be different. On the contrary, the vessels preserved in NSS with rhSLPI for 24 hours showed a significantly lower necrosis score when compared to the other groups (Fig. 4C). There were no significant differences in necrosis score between the vessels preserved in NSS with and without rhSLPI for 48 hours though there was a trend of reduction in necrosis score in the vessels preserved in rhSLPI group.

Similarly, the score for endothelial degeneration/edema in the vessels preserved for 6 hours showed that the vessels preserved in NSS with rhSLPI had a significantly lower endothelial degeneration/edema score when compared to the vessels preserved in NSS (Fig. 4E). However, the score of complete denudations showed no significant difference in any group (Fig. 4F).

\section{DISCUSSION}

Among crucial factors that affect the success of vascular reconstruction, the quality of vessel grafting, primarily influenced by the graft preservative/storage solution, influences the preservation of the endothelial structure and vascular functions (Woodward et al., 2016).

NSS is the most widely used preservative solution in vascular operative procedures (Kazemi et al., 2018; Weiss et al., 2009) as it is convenient and cheap. However, several studies have suggested unsatisfactory outcomes of its use, such as altered integrity of the endothelial layer and abolished vascular dilation (Weiss et al., 2009; Wilbring et al., 2011, Wilbring et al., 2013a, 2013b). Therefore, the preservative efficiency of NSS could be enhanced by adding supplements.

The previous studies have demonstrated that adding various substances such as heparin with papaverine (Santoli et al., 1993) or 5\% albumin (Weiss et al., 2009) could enhance the efficiency of NSS for vessel graft preservation. The previous reports have also shown that the cardiovascular-protective effect of rhSLPI could protect cardiomyocytes, cardiac fibroblast, and vascular endothelial cells from ischemia/reperfusion (I/R) injury (Nernpermpisooth et al., 2017; Paiyabhroma et al., 2018; Prompunt et al., 2018a, 2018b). Recently, we have demonstrated that endothelial-derived SLPI could protect cardiomyocytes from I/R injury (Kongpol et al., 2019), which suggests the beneficial effect of rhSLPI for cardiovascular applications. Interestingly, it has been shown that adding rhSLPI in a heart preservative solution could restore myocardial contraction during experimental murine heart transplantation (Schneeberger et al., 2008).

The major findings in the current study showed, for the first time, that rhSLPI could enhance the efficiency of conventional preservative NSS to preserve the isolated rat aortic vessels by reducing injury and inflammation and preserve the vascular structure. Furthermore, the experiments published by Schneeberger et al. were designed to test the effect of SLPI, either by gene knockout or treatment with recombinant protein, in transplanted murine hearts. The results showed that $200 \mu \mathrm{g}$ of recombinant SLPI given intravenously immediately after transplantation, or diluted in coldHTK preservation and flushed into the heart, could significantly improve a cardiac score, without the improvement of graft histology, and neither affected organ injury nor the inflammatory response. However, this study showed that only $1 \mu \mathrm{g} / \mathrm{ml}$ of rhSLPI (200 times less concentrate) dissolved in NSS could preserve vessel allograft by reducing tissue injury and inflammatory response and improve graft histology. Therefore, this study provides evidence that rhSLPI has the potential to be an alternative additive for vessel graft preservation.

The preservation in cold NSS could be affected by both profound low-storage temperature and bloodless-hypoxic conditions. These could induce biochemical and physiological stresses that are detrimental to vessel grafts and, consequently, 
Table 1. Nonscoring of vascular histopathology changes in aortic rings preserved in NSS or NSS with rhSLPI for $0,6,24$, and 48 hours.

\begin{tabular}{|c|c|c|c|c|}
\hline \multirow{2}{*}{\multicolumn{2}{|c|}{ Endothelial detachment }} & NSS (n) & NSS with rhSLPI $(n)$ & $p$-value \\
\hline & & & & \\
\hline \multirow[t]{2}{*}{ Control } & No change & 5 & - & \\
\hline & Change & 1 & - & \\
\hline \multirow[t]{2}{*}{6 hours } & No change & 1 & 5 & 0.080 \\
\hline & Change & 5 & 1 & \\
\hline \multirow[t]{2}{*}{24 hours } & No change & 1 & 5 & 0.080 \\
\hline & Change & 5 & 1 & \\
\hline \multirow[t]{2}{*}{48 hours } & No change & 3 & 2 & $>0.99$ \\
\hline & Change & 3 & 4 & \\
\hline \multicolumn{5}{|c|}{ Elastic membrane disruption } \\
\hline \multirow[t]{2}{*}{ Control } & No change & 5 & - & \\
\hline & Change & 1 & - & \\
\hline \multirow[t]{2}{*}{6 hours } & No change & 0 & 4 & 0.061 \\
\hline & Change & 6 & 2 & \\
\hline \multirow[t]{2}{*}{24 hours } & No change & 1 & 5 & 0.080 \\
\hline & Change & 5 & 1 & \\
\hline \multirow[t]{2}{*}{48 hours } & No change & 0 & 0 & $>0.99$ \\
\hline & Change & 6 & 6 & \\
\hline \multicolumn{5}{|l|}{ Necrosis } \\
\hline \multirow[t]{2}{*}{ Control } & No change & 6 & - & \\
\hline & Change & 0 & - & \\
\hline \multirow[t]{2}{*}{6 hours } & No change & 6 & 6 & $>0.99$ \\
\hline & Change & 0 & 0 & \\
\hline \multirow[t]{2}{*}{24 hours } & No change & 3 & 6 & 0.181 \\
\hline & Change & 3 & 0 & \\
\hline \multirow[t]{2}{*}{48 hours } & No change & 5 & 6 & $>0.99$ \\
\hline & Change & 1 & 0 & \\
\hline \multicolumn{5}{|c|}{ Endothelial degeneration/edema } \\
\hline \multirow[t]{2}{*}{ Control } & No change & 5 & - & \\
\hline & Change & 1 & - & \\
\hline \multirow[t]{2}{*}{6 hours } & No change & 2 & 5 & 0.242 \\
\hline & Change & 4 & 1 & \\
\hline \multirow[t]{2}{*}{24 hours } & No change & 5 & 6 & $>0.99$ \\
\hline & Change & 1 & 0 & \\
\hline \multirow[t]{2}{*}{48 hours } & No change & 6 & 6 & $>0.99$ \\
\hline & Change & 0 & 0 & \\
\hline \multicolumn{5}{|c|}{ Complete denudation } \\
\hline \multirow[t]{2}{*}{ Control } & No change & 6 & - & \\
\hline & Change & 0 & - & \\
\hline \multirow[t]{2}{*}{6 hours } & No change & 6 & 6 & $>0.99$ \\
\hline & Change & 0 & 0 & \\
\hline \multirow[t]{2}{*}{24 hours } & No change & 5 & 6 & $>0.99$ \\
\hline & Change & 1 & 0 & \\
\hline 48 hours & No change & 5 & 5 & $>0.99$ \\
\hline & Change & 1 & 1 & \\
\hline
\end{tabular}

cause tissue injury that eventually leads to graft failure. The determination of cellular injury could be confirmed by the detection of released (LDH - a cytosolic enzyme present in almost all cell types in the vasculature) activity. When the plasma membrane is ruptured or damaged, LDH is rapidly released into the surrounding environment (Chan et al., 2013; Kumar et al., 2018). Therefore, the determination of LDH activity is a marker of cellular injury. The vessels preserved in NSS with rhSLPI showed a lower released LDH activity (Fig. 1). This result indicated that

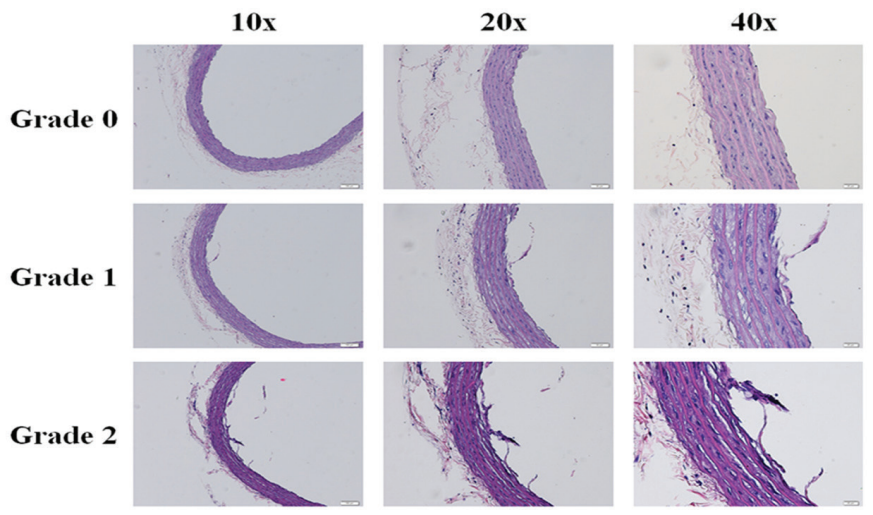

Figure 3. Scoring scheme of vascular histopathology. Aortic rings were preserved in $0.9 \%$ NSS or $0.9 \%$ NSS with rhSLPI preservative solution for 0,6 , 24 , and 48 hours. The vessel sections were stained with hematoxylin and eosin (H\&E) for histopathology analysis by a pathologist.

rhSLPI could be an alternative additive of choice supplement to NSS by reducing vessel graft injury during storage.

A short episode of ischemia/reperfusion (I/R) injury occurs during the isolation of vessel grafting and the transplantation procedure, respectively (Kazemi et al., 2018; Schneeberger et al., 2008). The I/R injury is an inflammatory process that increases the expression of pro-inflammatory cytokines, particularly the tumor necrosis factor $\alpha$ (TNF- $\alpha$ ) and interleukin-6 (IL-6), that cause cellular injury and death and induce leukocyte recruitment and platelet adhesion, which could lead to vascular occlusion and rejection after revascularization. These affect the success of organ transplants (Eltzschig and Eckle, 2011; Epelman et al., 2015; Kazemi et al., 2018). Moreover, ischemic injury could also induce a tissue inflammation by releasing pro-inflammatory cytokines (Yang et al., 2016).

One of the well-known functions of SLPI is antiinflammation (Doumas et al., 2005), which corresponds to these findings and demonstrated that vessels preserved in NSS with rhSLPI had less TNF- $\alpha$ and IL-6 levels than control (Fig. 2). Similar to the previous study, the pretreatment of rhSLPI in adipocytes indicated the downregulation of LPS-induced IL-6 gene expression and protein secretion in adipocytes (Adapala et al., 2011). Besides, mice hearts preserved in cold preservative with rhSLPI also decreased pro-inflammatory cytokines expression, including TNF- $\alpha$, Transforming growth factor (TGF)- $\beta$, Nuclear Factor Kappa B (NF-kB), eNOS, Potassium chloride (KCl) (Schneeberger et al., 2008). Therefore, adding rhSLPI to cold preservative could reduce pro-inflammatory cytokine levels, leading to a decreased inflammation of vessel grafts.

The previous studies have shown that NSS is an acceptable preservative although with limitations when compared to other preservative solutions (Kazemi et al., 2018; Zatschler et al., 2009). For example, the determination of preservation-induced changes in smooth muscle cell and ex vivo endothelial cell function in isolated porcine carotid arteries showed that NSS is the worst preservative solution, causing failure in the contraction and relaxation of the carotid artery, when compared to other preservatives, such as UW, HTK, Celsior, or a modified HTK solution (Abrahamse et al., 2002). Besides, a comparison between HTK and physiological saline solution (PSS) or the newly developed preservation solutions (solution 8 and solution 9) in isolated rat aorta showed that PSS 
A

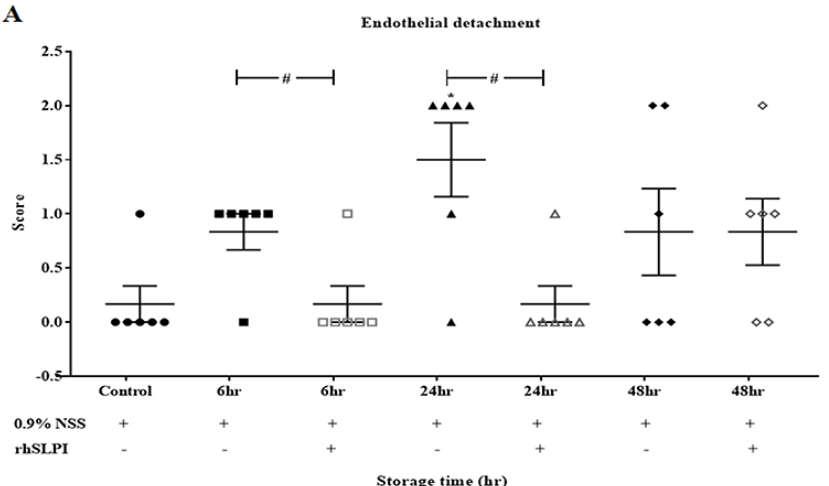

B

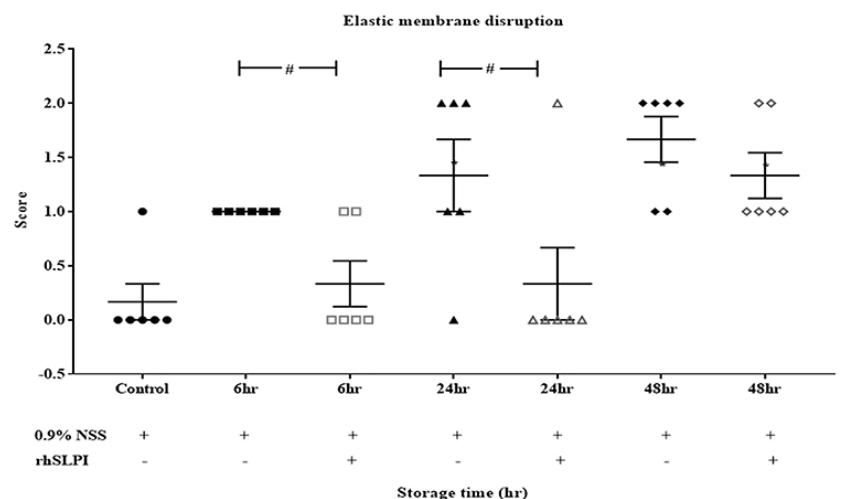

C

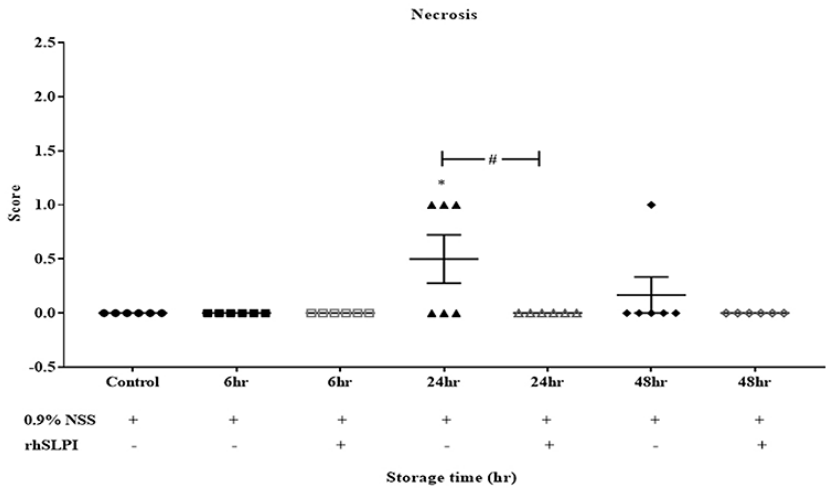

$\mathbf{D}$

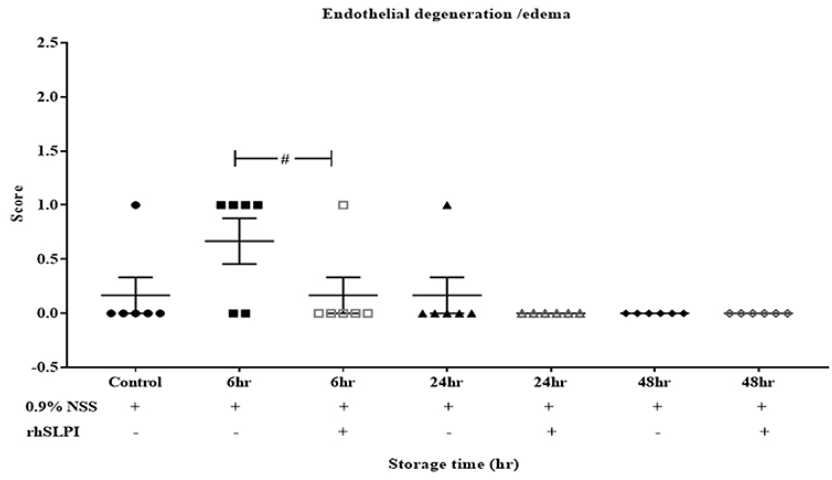

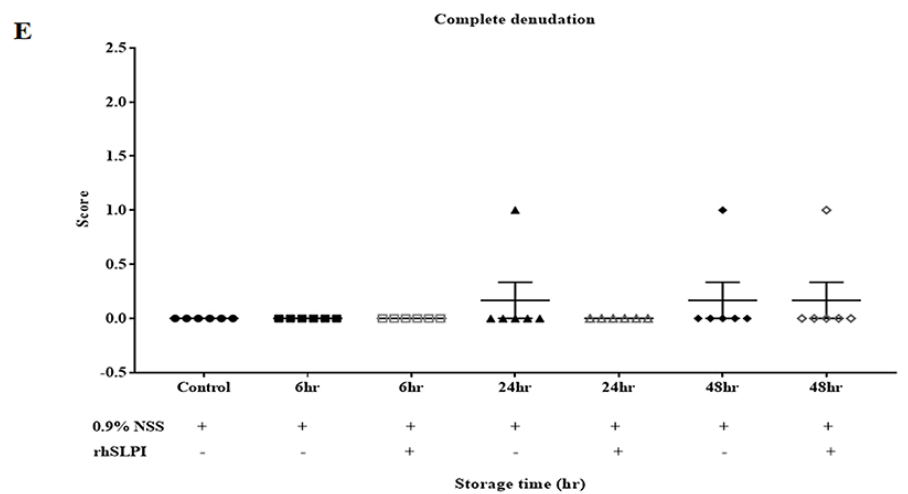

Figure 4. Vascular histopathology score after storage of aortic rings in $0.9 \%$ NSS in the presence and absence of rhSLPI for $0,6,24$, and 48 hours. The vascular histopathology parameters consisted of (A) endothelial detachment, (B) elastic membrane disruption, (C) necrosis, (D) endothelial degeneration/edema, and (E) complete denudation. Data are presented in a scatter plot with the values of means \pm S.E.M. ${ }^{*} p<0.05$ versus storage at 0 hour (ANOVA), $\# p<0.05$ versus among storage in $0.9 \%$ NSS, with or without rhSLPI (ANOVA, $n=6$ ).

failed to develop the vascular relaxation in isometric tension and endothelial nitric oxide synthase (eNOS) $\mathrm{KCl}$ expression (Zatschler et al., 2009). Furthermore, human saphenous vein preserved in NSS showed a reduction of $\mathrm{KCl}$ - and phenylephrine-induced vascular contraction, as well as endothelial-dependent and independent relaxation and cell viability when compared to UW solution, Celsior solution, and autologous whole blood (Wise et al., 2015). However, the aspect of preservation on the vascular structure by measuring vascular histopathology was not considered as there was insufficient information. In the previous study, isolated human femoral and iliac arteries preserved in NSS or UW solutions were assessed for vascular integrity changes using the histopathology technique on the 1st, 5th, 10th, and 21st days (Kazemi et al., 2018). The study demonstrated that there was no statistical difference in vascular pathological score between NSS and UW groups until 21st day (Kazemi et al., 2018). This was similar to the findings, indicating that there was no significant difference in the complete denudation score for the vessels preserved in NSS in the presence and absence of rhSLPI for 48 hours. However, the preservation for 24 hours resulted in a lower pathological degenerative score, particularly on endothelial detachment, elastic membrane disruption, and necrosis in NSS supplemented with rhSLPI (Table 1).

Interestingly, the histopathological score (Fig. 4A, C, and D) showed discrepancies in histopathological changes between 24 and 48 hours. It has been reviewed that the earliest event of vascular injury is endothelial edema, followed by endothelial 
necrosis, resulting in endothelial detachment (Woywodt et al., 2002). Therefore, at 6 hours after storage, we assumed that most endothelial cells were in the edema stage, which reflect why the scoring of endothelial edema at 6 hours was the highest (Fig. 4D). When the injury progressed between 24 and 48 hours, the necrosis of endothelial cells predominated, and therefore, the score of endothelial necrosis was observed as higher, whereas that of edema was lower (Fig. 4C). At 24-48 hours, endothelial detachment occurs (Fig. 4A), where endothelial detachment scores of both 24 and 48 hours predominated, without significant difference between 24 and 48 hours. At 48 hours, endothelial cell detachment occurs, and in particular, necrotic cells also disappeared from vascular tissue; therefore, the score of necrosis at 48 hours was observed less. This result provided novel information concerning the addition of rhSLPI into NSS to protect the vascular structure and could potentially increase the success rate of vascular transplantation.

There are several limitations that could be considered in this study, as follows: first, only the vascular structure was measured by vascular histopathological assessment. However, the effect of preservation with rhSLPI on vascular physiological functions, by using isometric tension, needs to be investigated. Moreover, the expression of adhesion molecules such as cell adhesion molecules, P-selectins, and integrin on activated endothelial cells should be investigated further to evaluate the risk of vessel graft failure under rhSLPI supplement in NSS. This experimental study performed a preclinical research using rats. The sample size $(n=6)$ was calculated as appropriate by a statistician with the awareness of unnecessary wastage of resources, which lead to ethical issues. However, the sample size in this study might only be appropriate for a laboratory study and might not provide more clinical significance. Therefore, we suggest using a larger sample size in future published articles, mostly in clinical studies of human samples.

For the first time, this study showed that rhSLPI could enhance the efficiency of NSS as a vascular preservative solution. However, the experimental perspective should focus on the effect of rhSLPI on vessel preservation in an in vivo animal model of transplantation. To closely simulate real clinical settings, the clinical outcome of vessel patency post-transplantation of vessel grafts, preserved in a preservative solution supplemented with rhSLPI, should be determined. This could be performed using angiography to determine the vascular parameters such as wall thickness and lumen diameter, as well as atherosclerotic lesions. From a clinical perspective, the outcomes from this study could be implemented for human vessel grafting and other organ transplants, and the long-term effects of transplanted grafts preserved in preservative solution, supplemented with rhSLPI, should be determined. Furthermore, the effect of rhSLPI in a preservative solution for other vital organs, such as the kidneys, brain, and liver, should be considered for further study.

\section{CONCLUSION}

The supplementation of vascular preservative solution with recombinant rhSLPI could reduce vascular injury and inflammation while maintaining vascular integrity.

For the first time, the results have shown that adding rhSLPI to the preservative saline solution could prevent vascular injury and possibly extend the duration of graft storage before transplantation.

\section{ACKNOWLEDGMENTS}

The authors would like to thank Assistant Professor Dr. Katechan Jampachaisri for comments and suggestion on statistical analysis. The authors are grateful to the Centre for Animal Research, Naresuan University, for their excellent technical assistance and animal care. The authors would also like to thank ProofRead4Sure service to English proof reading and editing.

\section{AUTHORS' CONTRIBUTIONS}

Kantapich Kongpol, Nitirut Nernpermpisooth, and Sarawut Kumphune conceived and designed the experiments. Kantapich Kongpol and Rungrueang Yodsheewan performed the experiments. Kantapich Kongpol, Rungrueang Yodsheewan, and Sarawut Kumphune analyzed the data. Sarawut Kumphune and Nitirut Nernpermpisooth contributed reagents/materials/analysis tools. Kantapich Kongpol, Nitirut Nernpermpisooth, and Sarawut Kumphune wrote and prepared the manuscript. Kantapich Kongpol, Rungrueang Yodsheewan, Nitirut Nernpermpisooth, and Sarawut Kumphune corrected, revived, commented, and responded to reviewer's comments.

\section{CONFLICTS OF INTEREST}

The authors declare no conflicts of interest.

\section{FINANCIAL SUPPORT AND SPONSORSHIP}

This work was supported by The Royal Golden Jubilee PhD Program [grant number PHD/0125/2558] for Kantapich Kongpol, Thailand Research Fund (RSA6280025) for Sarawut Kumphune, Naresuan University Endowment Fund [grant no.R2559C007] for Nitirut Nernpermpisooth

\section{REFERENCES}

Abrahamse STL, Dinant S, Pfaffendorf M, Van Gulik TM. In vitro function of porcine carotid arteries preserved in UW, HTK and Celsior solutions. Fundam Clin Pharmacol, 2002; 16:503-11.

Adapala VJ, Buhman KK, Ajuwon KM. Novel anti-inflammatory role of SLPI in adipose tissue and its regulation by high fat diet. J Inflamm (Lond), 2011; 8:5-5.

Ben Ali W, Voisine P, Olsen PS, Jeanmart H, Noiseux N, Goeken T, Satishchandran V, Cademartiri F, Cutter G, Veerasingam D, Brown C, Emmert MY, Perrault LP. DuraGraft vascular conduit preservation solution in patients undergoing coronary artery bypass grafting: rationale and design of a within-patient randomised multicentre trial. Open Heart, 2018; 5:e00780.

Chan FK-M, Moriwaki K, De Rosa MJ. Detection of necrosis by release of lactate dehydrogenase activity. Methods Mol Biol, 2013; 979:65-70.

Doumas S, Kolokotronis A, Stefanopoulos P. Anti-inflammatory and antimicrobial roles of secretory leukocyte protease inhibitor. Infect Immun, 2005; 73:1271-4.

Eltzschig HK, Eckle T. Ischemia and reperfusion-from mechanism to translation. Nat Med, 2011; 17:1391-401.

Epelman S, Liu PP, Mann DL. Role of innate and adaptive immune mechanisms in cardiac injury and repair. Nat Rev Immunol, 2015; 15:117-29.

Fahner PJ, Idu MM, van Gulik TM, Legemate DA. Systematic review of preservation methods and clinical outcome of infrainguinal vascular allografts. J Vasc Surg, 2006; 44:518-24.

Howat WJ, Wilson BA. Tissue fixation and the effect of molecular fixatives on downstream staining procedures. Methods, 2014; $70: 12-19$. 
Kazemi K, Nikeghbalian Z, Yaghmaei S, Nikeghbalian S, Shamsaeifar A, Asgharnia Y, Dehghankhalili M, Golchini A, Malekhosseini SA. University of Wisconsin vs normal saline solutions for preservation of blood vessels of brain dead donors: a histopathological study. Clin Transplant, 2018; 32:e13241.

Kieffer E, Gomes D, Chiche L, Fléron M-H, Koskas F, Bahnini A. Allograft replacement for infrarenal aortic graft infection: early and late results in 179 patients. J Vasc Surg, 2004; 39:1009-17.

Kongpol K, Nernpermpisooth N, Prompunt E, Kumphune S. Endothelial-cell-derived human secretory leukocyte protease inhibitor (SLPI) protects cardiomyocytes against ischemia/reperfusion injury. Biomolecules, 2019; 9:678.

Kumar P, Nagarajan A, Uchil PD. Analysis of cell viability by the lactate dehydrogenase assay. Cold Spring Harb Protoc, 2018; 2018(6). doi: 10.1101/pdb.prot095497.

Majchrzak-Gorecka M, Majewski P, Grygier B, Murzyn K, Cichy J. Secretory leukocyte protease inhibitor (SLPI), a multifunctional protein in the host defense response. Cytokine Growth Factor Rev, 2016; 28:79-93.

Mongkolpathumrat P, Nokkaew N, Adulyaritthikul P, Kongpol K, Sanit J, Pankhong P, Kumphune S. Diabetes induced internal organs inflammation in non-obese type 2 diabetic rats. J Appl Pharm Sci, 2019; 9:041-9.

Nernpermpisooth N, Prompunt E, Kumphune S. An in vitro endothelial cell protective effect of secretory leukocyte protease inhibitor against simulated ischaemia/reperfusion injury. Exp Ther Med, 2017; 14:5793-800

Paiyabhroma N, Nernpermpisooth N, Kumphune S. The recombinant human secretory leukocyte protease inhibitor (SLPI) protects cardiac fibroblasts injury against an in vitro ischemia/reperfusion injury. $\mathrm{J}$ Appl Pharm Sci, 2018; 8:156-62.

Prompunt E, Nernpermpisooth N, Sanit J, Kumphune S. Overexpression and pre-treatment of recombinant human secretory leukocyte protease inhibitor (rhSLPI) reduces an in vitro ischemia/ reperfusion injury in rat cardiac myoblast (H9c2) cell. Biomol Concepts, 2018a; 9:17-32.

Prompunt E, Sanit J, Barrère-Lemaire S, Nargeot J, Noordali $\mathrm{H}$, Madhani M, Kumphune S. The cardioprotective effects of secretory leukocyte protease inhibitor against myocardial ischemia/reperfusion injury. Exp Ther Med, 2018b; 15:5231-42.

Santoli E, Di Mattia D, Boldorini R, Mingoli A, Tosoni A, Santoli C. University of Wisconsin solution and human saphenous vein graft preservation: preliminary anatomic report. Eur J Cardiothorac Surg, $1993 ; 7: 548-52$.

Schneeberger S, Hautz T, Wahl SM, Brandacher G, Sucher R, Steinmassl O, Steinmassl P, Wright CD, Obrist P, Werner ER, Mark W, Troppmair J, Margreiter R, Amberger A. The effect of secretory leukocyte protease inhibitor (SLPI) on ischemia/reperfusion injury in cardiac transplantation. Am J Transplant, 2008; 8:773-82.
Weiss DR, Juchem G, Kemkes BM, Gansera B, Nees S. Extensive deendothelialization and thrombogenicity in routinely prepared vein grafts for coronary bypass operations: facts and remedy. Int J Clin Exp Med, 2009; 2:95-113.

Wilbring M, Ebner A, Schoenemann K, Knaut M, Tugtekin SM, Zatschler B, Waldow T, Alexiou K, Matschke K, Deussen A. Heparinized blood better preserves cellular energy charge and vascular functions of intraoperatively stored saphenous vein grafts in comparison to isotonic sodium-chloride-solution. Clin Hemorheol Microcirc, 2013a; 55:445-55.

Wilbring M, Tugtekin SM, Zatschler B, Ebner A, Reichenspurner H, Kappert U, Matschke K, Deussen A. Preservation of endothelial vascular function of saphenous vein grafts after long-time storage with a recently developed potassium-chloride and $\mathrm{N}$-acetylhistidine enriched storage solution. Thorac Cardiovasc Surg, 2013b; 61:656-62.

Wilbring M, Tugtekin SM, Zatschler B, Ebner A, Reichenspurner $\mathrm{H}$, Matschke K, Deussen A. Even short-time storage in physiological saline solution impairs endothelial vascular function of saphenous vein grafts. Eur J Cardiothorac Surg, 2011; 40:811-5.

Wille T, de Groot H, Rauen U. Improvement of the cold storage of blood vessels with a vascular preservation solution. Study in porcine aortic segments. J Vasc Surg, 2008; 47:422-31.

Wise ES, Hocking KM, Eagle S, Absi T, Komalavilas P, CheungFlynn J, Brophy CM. Preservation solution impacts physiologic function and cellular viability of human saphenous vein graft. Surgery, 2015; 158:537-46.

Woodward LC, Antoniades C, Taggart DP. Intraoperative vein graft preservation: what is the solution? Ann Thorac Surg, 2016; 102:173646.

Woywodt A, Bahlmann FH, de Groot K, Haller H, Haubitz M. Circulating endothelial cells: life, death, detachment and repair of the endothelial cell layer. Nephrol Dial Transplant, 2002; 17:1728-30.

Yang Q, He G-W, Underwood MJ, Yu C-M. Cellular and molecular mechanisms of endothelial ischemia/reperfusion injury: perspectives and implications for postischemic myocardial protection. Am J Transl Res, 2016; 8:765-777.

Zatschler B, Dieterich P, Müller B, Kasper M, Rauen U, Deussen A. Improved vessel preservation after 4 days of cold storage: experimental study in rat arteries. J Vasc Surg, 2009; 50:397-406.

How to cite this article:

Kongpol K, Yodsheewan R, Nernpermpisooth N, Kumphune $\mathrm{S}$. Recombinant human secretory leukocyte protease inhibitor (rhSLPI) ameliorated vessel preservation in experimentally isolated rat arteries. J Appl Pharm Sci, 2020; 10(09):107-114. 